\title{
A Novel Predictive Model for Anastomotic Leakage in Colorectal Cancer Using Auto-artificial Intelligence
}

\author{
JUNICHI MAZAKI, KENJI KATSUMATA, YUKI OHNO, RYUTARO UDO, TOMOYA TAGO, \\ KENTA KASAHARA, HIROSHI KUWABARA, MASANOBU ENOMOTO, \\ TETSUO ISHIZAKI, YUICHI NAGAKAWA and AKIHIKO TSUCHIDA
}

Department of Gastrointestinal and Pediatric Surgery, Tokyo Medical University, Tokyo, Japan

\begin{abstract}
Aim: Anastomotic leakage (AL) in left-sided colorectal cancer is a serious complication, with an incidence rate of $6-18 \%$. We developed a novel predictive model for AL in colorectal surgery with double-stapling technique (DST) anastomosis using auto-artificial intelligence (AI). Patients and Methods: A total of 256 patients who underwent curative surgery for left-sided colorectal cancer between 2017 and 2021 were included. In addition to conventional clinicopathological factors, we included the type of circular stapler using DST, conventional double-row circular stapler (DCS) or EEA ${ }^{T M}$ circular stapler with Tri-Staple ${ }^{T M}$ technology, $28 \mathrm{~mm}$ Medium/Thick (Covidien, New Haven, CT, USA) which had triple-row circular stapler (TCS) as a covariate. Auto-AI software Prediction One (Sony Network Communications Inc.) was used to predict $A L$ with 5-fold cross validation. Predictive accuracy was assessed using the area under the receiver operating characteristic curve. Prediction One also evaluated the 'importance of variables' (IOV) using a method based on permutation feature importance. Results: The area under the curve of the AI model was 0.766. The type of circular stapler used was the most influential factor contributing to AL (IOV=0.551). Conclusion: This auto-AI predictive model demonstrated an improvement in accuracy compared to the conventional model. It was suggested that use of a TCS may contribute to a reduction in the AL rate.
\end{abstract}

This article is freely accessible online.

Correspondence to: Junichi Mazaki, Department of Gastrointestinal and Pediatric Surgery, Tokyo Medical University, 6-7-1 Nishishinjyuku, Shinjyuku-ku, Tokyo 160-0023, Japan. Tel: +81 08333426111, e-mail: junichim@tokyo-med.ac.jp

Key Words: Artificial intelligence, predictive model, colorectal cancer, anastomotic leakage, auto-AI.
Colorectal cancer is the most common cancer globally and the second leading cause of cancer-related deaths, especially in Japan. Although for colorectal cancer anastomosis with the double-stapling technique (DST) using circular staplers is one of the most frequently employed standard methods (15 ), anastomotic leakage (AL) is a serious complication with an incidence rate of 6-18\% (6-10). AL affects not only shortterm outcomes, but also long-term oncological prognosis and functional outcomes (11). Successful anastomosis depends on several factors, including the tension between the two connected portions of the gastrointestinal tract, a healthy blood supply to the surrounding tissues, and the mechanical strength of the anastomosis (12-14).

Although many risk factors for AL have been reported, it remains difficult to accurately predict. Previous reports were analyzed using conventional statistical methods, such as multivariate analysis or nomograms (15-18). The accuracy of these retrospective reports was somewhat low at 0.75 . Recently, artificial intelligence (AI) has rapidly developed worldwide. With the development of sequencing technologies and computational methods to facilitate big data analysis, AI has been developed as a prognostic tool to refine precision and accuracy (19). Although it is difficult for clinical surgeons to develop machine-learning algorithms, the new auto machine-learning software Prediction One (Sony Network Communications Inc.) enables predictions to be evaluated quickly and easily.

The choice of surgical instruments for anastomosis greatly affects the safety of the operation. Conventionally, DST has been performed using a double-row circular stapler (DCS) but a new circular stapler, EEA ${ }^{\mathrm{TM}}$ circular stapler with Tri-Staple ${ }^{\mathrm{TM}}$ technology, $28 \mathrm{~mm}$ Medium/Thick (Covidien, New Haven, CT, USA) introduced recently has a triple row of staples and is expected to improve pressure resistance. However, the effectiveness of this new triple-row circular stapler (TCS) has not been fully verified or compared with conventional DST.

In this study, we aimed to develop a novel predictive model for AL of DST for colorectal cancer using auto-AI software Prediction One easily and quickly. 


\section{Patients and Methods}

Patients. Data from 256 patients who underwent curative surgery for left-sided colorectal surgery with DST anastomosis between January 2017 and October 2021 at Tokyo Medical University Hospital were acquired. The following 18 variables were extracted from this database: Age ( $\leq 75 />75$ years), sex, body mass index $\left(\leq 25 />25 \mathrm{~kg} / \mathrm{m}^{2}\right)$, American Society of Anesthesiologists score $(0 / \geq 1)$, preoperative hemoglobin ( $\mathrm{Hb})(<10 / \geq 10 \mathrm{~g} / \mathrm{dl})$, surgical procedure (open/minimum invasive surgery), diverting stoma (yes/no), normal/emergency surgery, operative time $(<400 / \geq 400 \mathrm{~min})$, hemorrhage $(<70 / \geq 70 \mathrm{ml})$, anastomotic level from anal verge (middle rectum, $5 \leq$ height $\leq 10 \mathrm{~cm} /$ upper rectum, $10<$ height $\leq 15 \mathrm{~cm}$ ), circular stapler used (DCS/TCS), postoperative complications (Clavien-Dindo classification >2), histological type (tub1/mucinous or signet/other), pathological T-status (pT), pathological N-status ( $\mathrm{pN}$ ) (negative/positive), lymphatic invasion (negative/positive), and venous invasion (negative/positive). We excluded patients who had anastomosis $<5 \mathrm{~cm}$ from the anal verge and who underwent preoperative therapy. Generally, patients were admitted to our hospital a few days before surgery, and we obtained laboratory values on the day of admission. This study was conducted in accordance with the guidelines of the Declaration of Helsinki. The Review Board of the Tokyo Medical University Hospital approved the study (T2019-0060), and informed consent was obtained from all patients.

Surgical treatment. We performed curative surgeries for all the patients. The same group of dedicated colorectal surgeons performed all surgeries. The anal side of the colorectum was incised using a linear stapler. The anvil of the circular stapler was secured in place, and end-to-end anastomosis was performed using the DST. The rod of the circular stapler was inserted from the opposite side of the linear staple line, piercing the rectal stump near the linear staple line.

Postoperative care for AL. The 30-day AL rate was recorded and cases were diagnosed according to the International Study Group of Rectal Cancer AL definition (20) as a defect of the intestinal wall at the anastomotic site, which leads to a communication between the intra- and extraluminal compartments, or as an abscess adjacent to the anastomosis. A computed tomographic (CT) scan with rectal contrast was performed in patients with suspected AL in the absence of unquestionable clinical signs of peritonitis, which would indicate urgent surgery. On postoperative days 3,5 , and 7 , the C-reactive protein level was determined in all cases. For patients with a Creactive protein level $>200 \mathrm{mg} / \mathrm{l}$, a CT scan was performed. The following CT findings were considered suggestive of anastomotic failure: Contrast leakage from inside the bowel to the pelvis or abdominal cavity, abscess, or perianastomotic collection associated with or without localized pneumoperitoneum.

Statistical analysis. Descriptive statistics are provided for all variables. Regarding anastomotic leakage, we set the cut-off values of each covariate using receiver operating curves. The relationship between the qualitative variables was established using the chisquare test. All statistical analyses were performed using EZR (Saitama Medical Center, Jichi Medical University, Saitama, Japan), which is a graphical user interface for R (The R Foundation for Statistical Computing, Vienna, Austria). The level of statistical significance was set at $p<0.05$.
Artificial intelligence. We used Prediction One (https:// predictionone.sony.biz; Sony Network Communications Inc.) machine-learning software to predict AL. The software generates feature vectors from the dataset using standard preprocessing methods, such as one-hot encoding for categorical variables and normalization for numerical variables. A gradient-boosting tree and a neural network are used as supervised machine-learning models, each trained with hyperparameter tuning. An ensemble model of both trained models was constructed. Missing values are automatically handled with common machine-learning techniques, such as using a gradient-boosting tree, which can handle missing values. The area under the receiver operating characteristics curve (AUC) was calculated using internal validation to evaluate the accuracy of the AI model. Prediction One made the best predictive model using an artificial neural network with 5-fold crossvalidation. Prediction One also evaluated the 'importance of variables' (IOV) using a method based on permutation feature importance. This method was used to calculate the difference in the model output when a single variable was removed. The value of the difference in the model output indicates how much the model depends on the variable. The value of the difference was computed for each covariate and then averaged over those in the dataset.

\section{Results}

Patient and tumor characteristics. Baseline patient and tumor characteristics of the entire cohort, divided by the presence or absence of AL are shown in Table I. The rate of AL for the entire cohort was $6 \%$. The rates of AL in the DCS and TCS subgroups were $7.7 \%$ and $2.7 \%$, respectively $(p=0.226)$.

AI analysis. We used Prediction One to analyze the model for predicting AL. Data from 156 and 100 patients were used for the learning and validation models, respectively. The receiver operating characteristics curve of the AI model is shown in Figure 1. The AUC was 0.766. Prediction One was also used to calculate the IOV of each factor for anastomotic leakage. Factors with IOV $\geq 0.040$, in decreasing order, were the type of circular stapler used (0.055), pT status $(0.053), \mathrm{pN}$ status (0.051), and venous invasion (0.043). The total IOV and IOV for AL are shown in Table II, with the distribution according to the presence and absence of AL. The contributions of DCS and pT status to AL were the highest among all covariates.

\section{Discussion}

The AL rate is on a downward trend compared to before, but it has not yet reached zero $(21,22)$. The strength of this study is that a clinical surgeon not familiar with the use of AI was able to construct a highly accurate predictive model using simple auto-AI. Prediction One can automatically produce the predictive model within a few minutes, as long as the dataset is provided. We developed a novel auto-AI-generated model for predicting AL in colorectal cancer surgery with high accuracy. We also included a new type of circular stapler as covariate to be considered as an important factor in AL. 
Table I. Baseline patient and tumor characteristics.

\begin{tabular}{|c|c|c|c|c|c|}
\hline \multirow[t]{2}{*}{ Factor } & & \multirow{2}{*}{$\begin{array}{l}\text { Overall, n (\%) } \\
\qquad(\mathrm{n}=256)\end{array}$} & \multicolumn{2}{|c|}{ Anastomotic leakage, n (\%) } & \multirow[b]{2}{*}{$p$-Value } \\
\hline & & & No $(n=240)$ & Yes $(n=16)$ & \\
\hline \multirow[t]{2}{*}{ Age } & $\leq 75$ Years & $182(71.1)$ & $169(70.4)$ & $13(81.2)$ & 0.522 \\
\hline & $>75$ Years & $74(28.9)$ & $71(29.6)$ & $3(18.8)$ & \\
\hline \multirow[t]{2}{*}{ Gender } & Male & $140(54.7)$ & $129(53.8)$ & $11(68.8)$ & 0.364 \\
\hline & Female & $116(45.3)$ & $111(46.2)$ & $5(31.2)$ & \\
\hline \multirow[t]{2}{*}{ BMI } & $\leq 25 \mathrm{~kg} / \mathrm{m}^{2}$ & $191(74.6)$ & $179(74.6)$ & $12(75.0)$ & $>0.99$ \\
\hline & $>25 \mathrm{~kg} / \mathrm{m}^{2}$ & $65(25.4)$ & $61(25.4)$ & $4(25.0)$ & \\
\hline \multirow[t]{2}{*}{ ASA score } & 0 & $107(41.8)$ & $103(42.9)$ & $4(25.0)$ & 0.252 \\
\hline & $\geq 1$ & $149(58.2)$ & $137(57.1)$ & $12(75.0)$ & \\
\hline \multirow[t]{2}{*}{ Preoperative $\mathrm{Hb}$} & $\geq 10 \mathrm{~g} / \mathrm{dl}$ & $116(45.3)$ & $106(44.2)$ & $10(62.5)$ & 0.356 \\
\hline & $<10 \mathrm{~g} / \mathrm{dl}$ & $140(54.7)$ & $134(55.8)$ & $6(37.5)$ & \\
\hline \multirow[t]{2}{*}{ Emergency } & No & $252(99.6)$ & $236(99.6)$ & $16(100.0)$ & 0.934 \\
\hline & Yes & $1(0.4)$ & $1(0.4)$ & $0(0.0)$ & \\
\hline \multirow[t]{2}{*}{ Procedure } & Open & $30(11.8)$ & $28(11.7)$ & $2(12.5)$ & $>0.99$ \\
\hline & MIS & $225(88.2)$ & $211(88.3)$ & $14(87.5)$ & \\
\hline \multirow[t]{2}{*}{ Operative time } & $<400 \min$ & $213(83.2)$ & $205(85.4)$ & $8(50.0)$ & 0.001 \\
\hline & $\geq 400 \mathrm{~min}$ & $43(16.8)$ & $35(14.6)$ & $8(50.0)$ & \\
\hline \multirow[t]{2}{*}{ Hemorrhage } & $<70 \mathrm{ml}$ & $175(68.4)$ & $170(70.8)$ & $5(31.2)$ & 0.003 \\
\hline & $\geq 70 \mathrm{ml}$ & $81(31.6)$ & $70(29.2)$ & $11(68.8)$ & \\
\hline \multirow[t]{2}{*}{ Anastomosis level } & Middle rectum $(5 \leq$ height $\leq 10 \mathrm{~cm})$ & $83(32.4)$ & $72(30.0)$ & $11(68.8)$ & 0.003 \\
\hline & Upper rectum $(10<$ height $\leq 15 \mathrm{~cm})$ & $173(67.6)$ & $168(70.0)$ & $5(31.2)$ & \\
\hline \multirow[t]{2}{*}{ Circular stapler } & DCS & $182(71.1)$ & $168(70.0)$ & $14(87.5)$ & 0.226 \\
\hline & TCS & $74(28.9)$ & $72(30.0)$ & $2(12.5)$ & \\
\hline \multirow[t]{2}{*}{ Diverting stoma } & No & $209(82.0)$ & $199(83.3)$ & $10(62.5)$ & 0.079 \\
\hline & Yes & $46(18.0)$ & $40(16.7)$ & $6(37.5)$ & \\
\hline \multirow[t]{4}{*}{ pT-stage } & 1 & $49(31.8)$ & $47(32.2)$ & $2(25.0)$ & 0.839 \\
\hline & 2 & $19(12.3)$ & $18(12.3)$ & $1(12.5)$ & \\
\hline & 3 & $53(34.4)$ & $51(34.9)$ & $2(25.0)$ & \\
\hline & 4 & $33(21.4)$ & $30(20.5)$ & $3(37.5)$ & \\
\hline \multirow[t]{2}{*}{ pN stage } & Negative & $145(60.7)$ & $136(61.0)$ & $9(56.2)$ & 0.913 \\
\hline & Positive & $94(39.3)$ & $87(39.0)$ & $7(43.8)$ & \\
\hline \multirow[t]{2}{*}{ Lymphatic invasion } & Negative & $104(43.7)$ & $96(43.2)$ & $8(50.0)$ & 0.791 \\
\hline & Positive & $134(56.3)$ & $126(56.8)$ & $8(50.0)$ & \\
\hline \multirow[t]{2}{*}{ Venous invasion } & Negative & $82(34.5)$ & $79(35.6)$ & $3(18.8)$ & 0.273 \\
\hline & Positive & $156(65.5)$ & $143(64.4)$ & $13(81.2)$ & \\
\hline \multirow[t]{3}{*}{ Histological type } & Tub1 & $58(24.5)$ & $54(24.4)$ & $4(25.0)$ & 0.485 \\
\hline & Other & $174(73.4)$ & $163(73.8)$ & $11(68.8)$ & \\
\hline & Muc/sig & $5(2.1)$ & $4(1.8)$ & $1(6.2)$ & \\
\hline \multirow[t]{2}{*}{ Postoperative complication ${ }^{\dagger}$} & No & $229(89.5)$ & $214(89.2)$ & $15(93.8)$ & 0.875 \\
\hline & Yes & $27(10.5)$ & $26(10.8)$ & $1(6.2)$ & \\
\hline \multirow[t]{2}{*}{ Anastomotic leakage } & No & $240(93.8)$ & & & \\
\hline & Yes & $16(6.2)$ & & & \\
\hline
\end{tabular}

ASA: American Society of Anesthesiologists; BMI: body mass index; DCS: double-row circular stapler; Hb: hemoglobin; MIS: minimally invasive surgery; muc: mucinous cell carcinoma; pN-stage: pathological N-stage; pT-stage: pathological T-stage; sig: signet ring cell carcinoma; TCS: triplerow circular stapler. ${ }^{\dagger}$ Clavien-Dindo classification $>2$. Statistically significant $p$-values are shown in bold.

Regarding the accuracy of previously reported models using conventional statistical analyses, reported values of the AUC were all under 0.75 (15-18). In the present study, the AUC of the AI model was 0.766, which is an acceptable value. Moreover, this model, which consists of simple clinicopathological factors that are easy to obtain, can be constructed at a low cost. The use of machine learning, which includes supervised or unsupervised approaches, is becoming more and more necessary in the field of clinical research. Conventional statistical analysis uses a (generalized) linear model that does not combine features. However, Prediction One uses a non-linear model (gradient-boosting tree and a neural network) that can handle combinations of features. This may be the reason for the improved accuracy. 


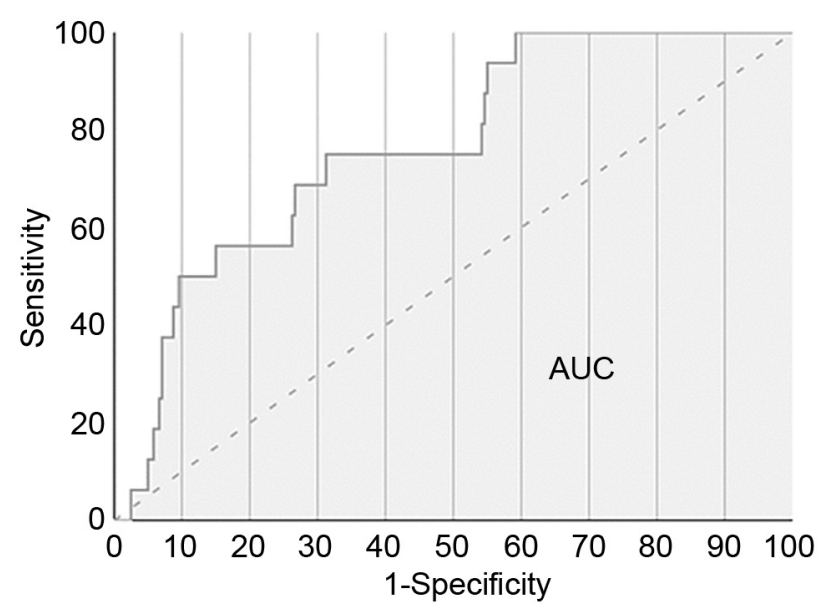

Figure 1. Receiver operating characteristic curve of the artificial intelligence model for anastomotic leakage. The area under the curve (AUC) was 0.766

Previously, we used a DCS for the DST in colorectal surgery. Since the EEA $^{\mathrm{TM}}$ circular stapler, a new circular stapler with TCS was introduced in Japan in January 2021, this device has been used to perform DST at our Institute. TCS with a triple row of staples is expected to improve pressure resistance, as compared to conventional DCS. However, the effectiveness of TCS in reducing the risk of AL has not been fully examined. In the present study, we included the type of circular stapler used in the DST in AI analysis. Among the many factors, in addition to the oncological factors such as $\mathrm{pT}$ and $\mathrm{pN}$ status predicted before the analysis, we found that the type of circular stapler was the superior factor related to AL. This suggests that changing the circular stapler from DCS to TCS may result in a reduction in AL.

There are some limitations to the present study. Firstly, we used a single-center, retrospective design. Secondly, it is difficult to understand the internal mechanism of AI analysis ('black-box problem') (23), which is problematic because entrusting important decisions to a system that is difficult to explain itself presents obvious dangers. Although Prediction One partly resolved the 'black-box problem' by calculating IOVs, we were not able to completely exclude this problem in the present analyses. Thirdly, external validation was not performed. Although external validation was not enforced, a 5-fold cross-validation was enforced. Finally, the number of patients was small for the deep learning analysis. A prospective study with a large number of patients and external validation will be necessary to further improve the AI model.

In conclusion, we developed a novel AI-generated predictive model with improved accuracy compared to the conventional statistical analysis model for predicting AL in patients with colorectal cancer. TCS may contribute to a reduction in the $\mathrm{AL}$ rate.
Table II. The importance of variables for anastomotic leakage.

\begin{tabular}{lccc}
\hline & Total IOV & \multicolumn{2}{c}{ IOV for AL } \\
\cline { 3 - 4 } & & Distribution factor & IOV \\
\hline Age & 0.032 & $\leq 75$ Years & 0.012 \\
Gender & 0.014 & Male & 0.010 \\
BMI & 0.018 & $>25 \mathrm{~kg} / \mathrm{m}^{2}$ & 0.010 \\
ASA score & 0.026 & $\geq 1$ & 0.011 \\
Preoperative Hb & 0.037 & $\geq 10 \mathrm{~g} / \mathrm{dl}$ & 0.026 \\
Emergency & 0.020 & No & 0.012 \\
Procedure & 0.035 & MIS & 0.010 \\
Operative time & 0.027 & $\geq 400 \mathrm{~min}$ & 0.012 \\
Hemorrhage & 0.030 & $\geq 70 \mathrm{ml}$ & 0.017 \\
Anastomosis level & 0.036 & Middle rectum & 0.019 \\
& & $(5 \leq h e i g h t \leq 10 \mathrm{~cm})$ & \\
Circular stapler & $0.055^{*}$ & DCS & $0.037 *$ \\
Diverting stoma & 0.017 & No & 0.013 \\
Pathological T-status & 0.053 & T4 & $0.037^{*}$ \\
Pathological N-status & 0.051 & Negative & 0.030 \\
Lymphatic invasion & 0.026 & Negative & 0.011 \\
Venous invasion & 0.043 & Positive & 0.026 \\
Histological type & 0.025 & Muc/sig & 0.010 \\
Postoperative complication & & & \\
& 0.017 & No & 0.010 \\
\hline
\end{tabular}

AL: Anastomotic leakage; ASA: American Society of Anesthesiologists; BMI: body mass index; DCS: double-row circular stapler; $\mathrm{Hb}$ : hemoglobin; IOV: Importance of variable; MIS: minimally invasive surgery; muc: mucinous cell carcinoma; $\mathrm{pN}$-stage: pathological $\mathrm{N}$-stage; pT-stage: pathological T-stage; sig: signet ring cell carcinoma; TCS: triplerow circular stapler. ${ }^{\dagger}$ Clavien-Dindo classification $>2$. *The highest value.

\section{Conflicts of Interest}

The Authors declare that there are no conflicts of interest.

\section{Authors' Contributions}

J.M. wrote the main article and R.U., T.T., K.K., H.K., M.E. and T.I. acquired data for the work. KK, YN and AT drafted the work or revised it critically for important intellectual content. All Authors reviewed the final article.

\section{References}

1 Offodile AC 2nd, Feingold DL, Nasar A, Whelan RL and Arnell TD: High incidence of technical errors involving the EEA circular stapler: a single institution experience. J Am Coll Surg 210(3): 331335, 2010. PMID: 20193897. DOI: 10.1016/j.jamcollsurg. 2009.11.007

2 Slieker JC, Daams F, Mulder IM, Jeekel J and Lange JF: Systematic review of the technique of colorectal anastomosis. JAMA Surg 148(2): 190-201, 2013. PMID: 23426599. DOI: 10.1001/2013.jamasurg.33

3 Mirnezami R, Soares A and Chand M: Enhancing the precision of circular stapled colorectal anastomosis: could powered stapler technology provide the solution? Tech Coloproctol 
23(7): 687-689, 2019. PMID: 31278457. DOI: 10.1007/s10151019-02031-9

4 Choi HK, Law WL and Ho JW: Leakage after resection and intraperitoneal anastomosis for colorectal malignancy: analysis of risk factors. Dis Colon Rectum 49(11): 1719-1725, 2006. PMID: 17051321. DOI: 10.1007/s10350-006-0703-2

5 Veyrie N, Ata T, Muscari F, Couchard AC, Msika S, Hay JM, Fingerhut A, Dziri $\mathrm{C}$ and French Associations for Surgical Research: Anastomotic leakage after elective right versus left colectomy for cancer: prevalence and independent risk factors. J Am Coll Surg 205(6): 785-793, 2007. PMID: 18035262. DOI: 10.1016/j.jamcollsurg.2007.06.284

6 Karanjia ND, Corder AP, Bearn P and Heald RJ: Leakage from stapled low anastomosis after total mesorectal excision for carcinoma of the rectum. Br J Surg 81(8): 1224-1226, 1994. PMID: 7953369. DOI: 10.1002/bjs.1800810850

7 Cohen Z, Myers E, Langer B, Taylor B, Railton RH and Jamieson C: Double stapling technique for low anterior resection. Dis Colon Rectum 26(4): 231-235, 1983. PMID: 6839891. DOI: 10.1007/BF02562484

8 Jestin P, Påhlman L and Gunnarsson U: Risk factors for anastomotic leakage after rectal cancer surgery: a case-control study. Colorectal Dis 10(7): 715-721, 2008. PMID: 18318752. DOI: $10.1111 / \mathrm{j} .1463-1318.2007 .01466 . x$

9 Rullier E, Laurent C, Garrelon JL, Michel P, Saric J and Parneix M: Risk factors for anastomotic leakage after resection of rectal cancer. Br J Surg 85(3): 355-358, 1998. PMID: 9529492. DOI: 10.1046/j.1365-2168.1998.00615.x

10 Law WI, Chu KW, Ho JW and Chan CW: Risk factors for anastomotic leakage after low anterior resection with total mesorectal excision. Am J Surg 179(2): 92-96, 2000. PMID: 10773140. DOI: 10.1016/s0002-9610(00)00252-x

11 Kim CW, Baek SJ, Hur H, Min BS, Baik SH and Kim NK: Anastomotic leakage after low anterior resection for rectal cancer is different between minimally invasive surgery and open surgery. Ann Surg 263(1): 130-137, 2016. PMID: 25692355. DOI: $10.1097 /$ SLA.0000000000001157

12 Chung RS, Hitch DC and Armstrong DN: The role of tissue ischemia in the pathogenesis of anastomotic stricture. Surgery 104(5): 824-829, 1988. PMID: 3187897

13 Chung RS: Blood flow in colonic anastomoses. Effect of stapling and suturing. Ann Surg 206(3): 335-339, 1987. PMID: 3307654. DOI: $10.1097 / 00000658-198709000-00011$

14 Baker RS, Foote J, Kemmeter P, Brady R, Vroegop T and Serveld M: The science of stapling and leaks. Obes Surg 14(10): 12901298, 2004. PMID: 15603641. DOI: 10.1381/0960892042583888

15 Yao HH, Shao F, Huang Q, Wu Y, Qiang Zhu Z and Liang W: Nomogram to predict anastomotic leakage after laparoscopic anterior resection with intracorporeal rectal transection and double-stapling technique anastomosis for rectal cancer. Hepatogastroenterology 61(133): 1257-1261, 2014. PMID: 25436293.
16 Dekker JW, Liefers GJ, de Mol van Otterloo JC, Putter H and Tollenaar RA: Predicting the risk of anastomotic leakage in leftsided colorectal surgery using a colon leakage score. J Surg Res 166(1): e27-e34, 2011. PMID: 21195424. DOI: 10.1016/ j.jss.2010.11.004

17 Frasson M, Flor-Lorente B, Rodríguez JL, Granero-Castro P, Hervás D, Alvarez Rico MA, Brao MJ, Sánchez González JM, Garcia-Granero E and ANACO Study Group: Risk factors for anastomotic leak after colon resection for cancer: multivariate analysis and nomogram from a multicentric, prospective, national study with 3193 patients. Ann Surg 262(2): 321-330, 2015. PMID: 25361221. DOI: 10.1097/SLA.0000000000000973

$18 \mathrm{Kim}$ CH, Lee SY, Kim HR and Kim YJ: Nomogram prediction of anastomotic leakage and determination of an effective surgical strategy for reducing anastomotic leakage after laparoscopic rectal cancer surgery. Gastroenterol Res Pract 2017: 4510561, 2017. PMID: 28592967. DOI: $10.1155 / 2017 / 4510561$

19 Zhao M, Tang Y, Kim H and Hasegawa K: Machine learning with k-means dimensional reduction for predicting survival outcomes in patients with breast cancer. Cancer Inform 17: 1176935118810215 , 2018. PMID: 30455569. DOI: 10.1177/1176935118810215

20 Rahbari NN, Weitz J, Hohenberger W, Heald RJ, Moran B, Ulrich A, Holm T, Wong WD, Tiret E, Moriya Y, Laurberg S, den Dulk M, van de Velde C and Büchler MW: Definition and grading of anastomotic leakage following anterior resection of the rectum: a proposal by the International Study Group of Rectal Cancer. Surgery 147(3): 339-351, 2010. PMID: 20004450. DOI: 10.1016/j.surg.2009.10.012

21 Nagaoka T, Yamaguchi T, Nagasaki T, Akiyoshi T, Nagayama S, Fukunaga Y, Chino A, Ishizuka N and Konishi T: Safety of small circular staplers in double stapling technique anastomosis for sigmoid colon and rectal cancer. Dis Colon Rectum 64(8): 937-945, 2021. PMID: 33951685. DOI: 10.1097/DCR.0000000000001889

22 Ando K, Kuriyama N, Fujimoto Y, Jogo T, Hokonohara K, Hu Q, Hisamatsu Y, Nakanishi R, Nakashima Y, Kimura Y, Oki E and Mori M: New anastomosis technique to prevent anastomotic leakage in laparoscopic anterior resection for rectal cancer, especially upper rectal cancer. In Vivo 34(6): 3533-3538, 2020. PMID: 33144464. DOI: 10.21873/invivo.12195

23 LeCun Y, Bengio Y and Hinton G: Deep learning. Nature 521(7553): 436-444, 2015. PMID: 26017442. DOI: 10.1038/nature 14539
Received August 7, 2021

Revised October 7, 2021

Accepted October 12, 2021 